\title{
Employment of a Multi-measure Approach as a Vehicle for Monitoring Encoding and Retrieval Dynamics: The Case of Semantically Related Words
}

\author{
Jonathan Guez ${ }^{1}$, Moshe Naveh-Benjamin ${ }^{2}$ \\ ${ }^{1}$ Department of Behavioral Sciences, Achva Academic College, Achva, Israel; \\ ${ }^{2}$ Department of Psychological Sciences, University of Missouri, Columbia, U.S.A. \\ Email: jonjon@bgu.ac.il
Received April 18
th
2011; revised May 26 $6^{\text {th }}$, 2011; accepted July 1 ${ }^{\text {st }}, 2011$.
}

\begin{abstract}
Several recent studies have indicated that retrieval, unlike encoding, is only minimally affected by dividing attention, but is associated with significant secondary task costs. The reported experiment manipulated the strength of pre-experimental semantic associations, using a cued-recall paradigm and a multi-measure approach. This allowed the assessment of accuracy and latency of retrievals, as well as of overall attentional costs and the temporal distribution of attentional costs incurred by these retrievals. By simultaneously inspecting the different measures, we identified a common set of retrieval types and retrieval components. The results presented different patterns for semantically related or un-related words under full or divided attention as a function of the dependent measure used. The results emphasize the advantage of using multi-measure approach to the uncovering of different properties of cognitive processes.
\end{abstract}

Keywords: Divided Attention, Encoding, Retrieval, Semantic Relationships

\section{Introduction}

Earlier research on human memory suggested that the similarity between encoding and retrieval processes is an important factor in successful remembering. These lines of research include the encoding specificity principle (Tulving \& Thompson, 1973; Tulving, 1983), the transfer-appropriate processing view (Morris, Bransford \& Franks, 1977; Roediger, 1990; Roediger et al., 2002), and the proceduralist view of mind (Kolers, 1973); all carry the notion of a necessary overlap between encoding and retrieval processes and imply that experimental manipulations should have similar effects on encoding and retrieval. Neuroscientists (e.g., Danker \& Anderson, 2010; Mishkin \& Appenzeller, 1987; Moscovitch, 1992; Squire, 1992), have also suggested that the same neural pathways mediate the perceptual processing of stimuli and their storage and retrieval. In contrast, recent research using the divided attention (DA) paradigm has shown single dissociation between encoding and retrieval processes. When participants' attention is divided between encoding and a secondary task, memory performance is reduce compared to conditions when they pay full attention to encoding the items (e.g., Baddeley, Lewis, Eldrige \& Thomson, 1984; Craik, Govoni, Naveh-Benjamin, \& Anderson, 1996; Naveh-Benjamin, Craik \& Guez, 1998; Naveh-Benjamin, Craik, Gavrilescu \& Anderson, 2000; Naveh-Benjamin \& Guez, 2000). These results indicate that encoding processes require attention and that the allocation of attention to encoding processes is under the participant's conscious control. The reported effects of divided attention on retrieval processes are different. Participants in Baddeley et al. (1984), Craik et al. (1996), Naveh-Benjamin et al., (1998), Naveh-Benjamin,
Craik, Perretta \& Tonev (2000), Naveh-Benjamin \& Guez (2000), showed only small decreases in free recall, cued recall, and recognition performance, when attention was divided at retrieval. This relative immunity of memory to divided attention at retrieval was shown by Craik et al. (1996), and Naveh-Benjamin \& Guez (2000), and Naveh-Benjamin et al. (2000) to be accompanied by substantial secondary task reaction time costs which decreased from free recall to cued recall to recognition (see also Griffith, 1976; Johnston, Greenberg, Fisher, \& Martin, 1970; Johnston, Griffith \& Wagstaff, 1972). Furthermore, instructing the participants to emphasize the memory task, the secondary task, or both equally had no effect under DA at retrieval, but had a complementary effects on the two tasks under DA at encoding. As attention is switched to the secondary task and away from the memory task, memory performance declines and secondary task performance improves. Craik et al. and NavehBenjamin et al. therefore suggested that retrieval processes are in some sense obligatory, or protected, but that their execution requires substantial resources, as shown by the large secondary task costs. By contrast, encoding processes require attention but that allocation of attention to encoding processes is under the participant's conscious control. In a recent studies by us (Naveh-Benjamin \& Guez, 2000 and Guez \& Naveh-Benjamin, 2006), we employed a cued-recall task which allows the division of attentional resources required at retrieval into three retrieval phases or subcomponents: cue encoding, cue-elaboration/search processes for a specific word, and the operation of a retrieval mode. This was achieved by dividing each retrieval interval into three phases (partially based on Tulving's, 1983 taxonomy of retrieval sub-processes) and evaluating the gradient of attention 
required with each. The first phase includes the period until the end of the presentation of the cue, which presumably mostly consists of cue-encoding operations. The second phase, which consists of cue-elaboration/search processes, extends from the point at which a given cue is provided to the participant, until s/he furnishes a retrieved response. The demand for resources associated with cue-elaboration/search processes was differentiated from consumption of resources by a retrieval mode which can be evaluated during the period following a retrieval and before the next cue is presented. During this period, the participants are in a cognitive mode of retrieval readiness without a specific cue directing them to search for a particular target. For comparison purposes, the same time periods were designated to encoding. Participants' performance on the secondary tracking tasks gave us the precise micro-level measurement of those resources devoted to encoding and retrieval every $20 \mathrm{~ms}$. The results of both researches reported by Naveh-Benjamin \& Guez (2000) and Guez \& Naveh-Benjamin (2006) indicate that retrieval, at least during a cued-recall task, does not require uniform attentional resources. The first phase of cue-encoding requires some resources, much like the requirement for resources by stimuli detection at encoding. The second phase, involving cue-elaboration/search processes requires substantial resources for its execution. This was shown in two ways. First, for successful retrievals, the period after the cue-encoding and prior to the retrieved response was associated with poorer performance on the secondary task (larger tracking distance), than either the respective period at encoding or the retrieval period following the retrieval response. Second, the use of a cued-recall task allowed us to measure attentional resources associated with both successful and unsuccessful retrievals. Performance on the secondary task for unsuccessful retrievals (where no retrieved response was provided by the participant) was poor throughout the retrieval period of a given target. Specifically, while unsuccessful retrievals consume attentional resources for their execution as successful retrievals do up to the point of the retrieved response, these unsuccessful retrievals continue to require resources for the whole retrieval period, presumably reflecting the continuation of cue elaboration/search processes.

\section{Aims of the Current Study}

One purpose of the current study was to replicate and consolidate the above results reported by Naveh-Benjamin \& Guez (2000) and Guez \& Naveh-Benjamin (2006), since these are the only studies in the literature to report about attentional costs associated with specific sub-processes at retrieval. A second purpose was to extend the Naveh-Benjamin \& Guez' results. In particular, one interesting question is whether strengthening the encoded trace by the use of either semantic information will similarly affect the attentional costs associated with cue-elaboration/search process. Third, we wish to advocate the use of a multimeasure approach. In the current study, we used four measures of performance: memory accuracy, retrieval latency, overall attentional costs, and the temporal distribution of attentional costs. We believe that such an approach, the usefulness of which was demonstrated here, provides a more comprehensive perspective on the phenomenon under investigation.The claim that episodic retrieval is mediated and helped by semantic knowledge has been advocated time and again in the memory research (e.g., Herrmann \& McLaughlin, 1974; Bransford \& Johnson, 1972; Naveh-Benjamin, Craik \& Ben-Shaul, 2002). If this is the case, we expect that giving the participant a cue which is semantically related to the target, should enhance and help both the success of the search for the target as well as the ease in which this search is conducted. While previous research has shown that retrieval success is facilitated by semantic relatedness, very few studies to date have dealt with ease of access and no study has assessed the amount of attentional resources required for such access. In the few studies that assessed the ease of access (e.g., Dosher, 1984; Dosher \& Rosedale, 1991) a speed-accuracy tradeoff paradigm was used. These studies indicate that the pre-experimental (semantic) relationships between pairs of items can be used in addition to experimental (episodic) relationships, to enhance (speed up) the rate of retrieval of associative information. In the reported experiment, we manipulated the semantic relatedness of the cue-target pairs. We expected that related pairs would be retrieved faster and would also require fewer attentional resources, as participants can use their pre-experimental knowledge to access the target given the cue. Such a pattern should be especially noticeable during the cueelaboration/search phase. More specifically, several issues were investigated with the purpose of extending the results reported by Naveh-Benjamin \& Guez (2000) and Guez \& Naveh-Benjamin (2006) regarding the identifications of different types of retrievals. First, we wanted to validate the different patterns of attentional costs associated with successful and unsuccessful retrievals. As mentioned above, the authors showed that whereas both types of retrievals require the same amount of attentional resources for the execution of the cue-encoding and the cue-elaboration/search phases, the pattern of their attentional cost subsequently diverges: while successful retrievals (where the retrieval response terminates the cue-elaboration/search phase), are associated with fewer attentional resources, unsuccessful retrievals (where the cueelaboration/search phase proceeds until the next cue is presented) are associated with continuous high attentional costs. Using manipulations of pair-relatedness we wanted to evaluate the robustness of the differential pattern of attentional costs associated with successful and unsuccessful retrievals. In this context we may assessed whether successful retrievals would be less costly in terms of attentional resources if a presented cue is semantically pre-experimentally related to the target, or whether it would only affect retrieval success (increase the proportion of such retrievals).

Another goal of the current research was to evaluate the existence of different types of successful retrievals. NavehBenjamin \& Guez (2000) and Guez \& Naveh-Benjamin (2006) used a measure of retrieval latency to distinguish between two types of retrievals, and estimated the attentional resources associated with each. The results of their experiments indicated that these two types of retrievals require different amount of resources for their execution. One type, tentatively defined as strategic or algorithmic (see Guynn \& McDaniel, 1999; Logan, 1988), characterized by slow retrievals, was associated with substantial attentional resources for their execution, especially during the cue-elaboration/ 
search phase. The other type, tentatively defined as one-step or direct (see Guynn \& McDaniel, 1999; Logan, 1988), was characterized by fast retrievals (measured by a retrieval response within less than 1 safter the cue was presented). This type, comprised about $25 \%$ of all successful retrievals, required fewer resources for their execution, and in particular did not seem to show an increase in required attentional resources during the cue-elaboration/search phase. One interpretation of these results is that the one-step fast retrievals bypass cue-elaboration/search processes. Such a suggestion, which posits the operation of two qualitatively different types of successful retrievals - one which is fast and requires few attentional resources for its completion (one-step), and another which is slower and requires substantial resources (strategic) -- is in line with several suggestions in the literature. These suggestions depict the retrieval process as variable with different retrievals being based on different mechanisms: for example, Jacoby's distinction of familiarity and recollection (Jacoby, 1991), Gardiner's distinction between know and remember responses (Gardiner \& Java, 1993), and Logan's distinction between one step and algorithmic retrievals (Logan, 1988; see also Tzelgov, Yehene \& Naveh-Benjamin, 1997).

The manipulations of pair-relatedness used in the current studies, were intended to shed more light on these two types of successful retrievals. One question addressed in the current study was whether these retrievals, identified by the authors, can consistently be documented regardless of the manipulations used. A second question was whether and how these manipulations would affect the pattern of attentional costs required for the two types of successful retrievals. For example, would the use of semantic supportive conditions (semantically related pairs) change the pattern of attentional requirements, or would it only affect the proportion of each type of retrieval? Specifically, the use of related pairs could either decrease the attentional costs associated with strategic retrievals or merely decrease the proportion of this type of retrievals without changing the costs associated with them.

Finally, we wanted to assess whether the identification of different types of successful retrievals (strategic and one-step) made by the authors, which was based on participants' response latency criterion, can also be obtained when stimuli-based criterion is used. Specifically, the question asked was whether semantic manipulation, would also create two types of successful retrievals, strategic ones characterized by an attention-demanding cue-elaboration/search processes, and one-step ones, characterized by the absence of an attention-demanding cue-elaboration/search process.

To summarize, we assessed the secondary task costs associated with what seem to be obligatory retrieval processes, costs which are sometimes greater than those associated with encoding. Specifically, we wished to assess the costs associated with different phases of the retrieval period. We also wanted to differentiate between the attentional costs required by different types of retrievals (i.e., unsuccessful, strategic and one-step successful). To this end, we used well-established memory paradigms in which encoding and retrieval phases are clearly separated. To avoid modality-specific interference, we presented the verbal information to be remembered auditorily, and asked for spoken responses at re- trieval, while the concurrent tasks used visual stimuli and manual responses. We used a visual tracking task in which participants had to follow a fast-moving target on the computer monitor with a computer mouse. We employed a cued-recall task previously shown (e.g., Craik et al., 1996) to impose high demands for resources both at encoding and at retrieval, as well as a tracking procedure similar to the one used by Naveh-Benjamin \& Guez (2000) and Guez \& Naveh-Benjamin (2006) as the secondary task to be performed along with encoding or retrieval. This procedure allows a micro-level analysis of momentary changes in participants' performance by requiring participants to track a fast-moving target on a computer screen with a computer mouse. The program provides, in addition to an overall measure as previous studies did, a temporal distribution measure of performance, which is the spatial distance between the target and the tracker every $20 \mathrm{~ms}$ in a continuous fashion. The exact times when stimuli are presented auditorily by the experimenter during encoding (cue-target) and retrieval (cue), and of participants' vocal responses during retrieval (target), are recorded by the computer through the use of a voice-operated relay, and are superimposed on the continuous distance measure. This enables the measurement of the tracking task performance at virtually any moment during the encoding and retrieval phases. Because performance on either task performed singly did not reach ceiling, we contend that each task required full attention when performed alone. When performed together, the tasks allowed the assessment of performance throughout the dual-task interval. In particular, the secondary tracking task monitors and reflects the changes in attentional resources devoted to the encoding and retrieval of the words. We created two aggregated segments of the temporal distribution of attentional resources, one for encoding and one for retrieval. At encoding this was done by superimposing all 5-s segments, during which a word pair was presented, on top of each other, and measuring the average attentional cost gradient in these $5 \mathrm{sec}$. At retrieval, we superimposed all 5-s segments, where a cue was presented and a retrieved response was or was not provided, on top of each other (see Results section for details). In both cases, the aggregated 5-s single tracking task used as a baseline was created and superimposed, then subtracted from the encoding and retrieval aggregated segments at $50 \mathrm{~ms}$ slices. These segments provided us with a micro-level temporal distribution of the attentional resources required throughout the encoding and retrieval phases. We examined memory performance as well as the secondary tracking task performance to confirm that in such a paradigm the previously obtained differential memory effects of divided attention at encoding and retrieval are found. Finally, retrieval latency was measured as the time between the presentation of the cue and the initiation of the response as recorded by the voice-operated relay. This latency provided one criterion for the differentiation of different types of retrievals (i.e., slow vs. fast). Finally, as mentioned above, we manipulated the strength of the semantic relationships between the members of each pair with the intention of learning more abut characteristics of retrieval processes. 


\section{Method}

\section{Participants}

The participants, randomly assigned to this experiment, were 37 Ben-Gurion University of the Negev undergraduates, who took part in the experiment for course credit.

\section{Design}

Two independent within-subject variables were used. One was attention: either full attention, divided attention at encoding, or divided attention at retrieval. The other was the semantic relatedness of the pair (related vs. unrelated semantically). The dependent variables were proportion of correctly recalled targets, performance on the secondary tracking task, and retrieval latency.

\section{Stimuli}

The words used were high frequency two- or three-syllable concrete nouns taken from Hebrew norms (Balgur, 1968). Twelve lists were created, with 12 word pairs in each. Six of the lists contained unrelated word pairs and the other six lists contained related word pairs. The two words in each pair of the unrelated condition were semantically unrelated to each other. The two words in each pair in the related condition were semantically related, using Hebrew norms (Henik \& Kaplan, 1988). In both conditions there were no semantic relationships between words of different pairs or between words in the different lists. The A-B pairs were presented auditorily at study at a pace of one every $5 \mathrm{~s}$. At test the A word of each pair was presented as a cue and the participant had to produce the B response within 5-s (pilot work showed that there were very few responses after $5 \mathrm{sec}$ ). The tracking task involved a PC computer screen on which an asterisk moved at a rate of $6 \mathrm{~cm} / \mathrm{second}$ in a smooth continuous fashion. This rate was chosen in a pilot study as one which is moderately difficult for participants when used alone (their performance indicated no ceiling effect, as the distance measured was significantly higher than zero mm). Four tracking paths were designated, being combinations of left-right and up-down directions. Although the movement of the asterisk appeared to be random, it had been pre-designated for each path. Participants followed the asterisk with the computer mouse which controlled a plus(+) sign indicating their position on the screen, trying to stay as close as possible to the asterisk.

\section{Procedure}

Each participant was presented with the 12 lists consisting of two replications of all combinations of the three attention conditions and the two relatedness levels. In addition, each participant performed the tracking baseline task four times, each time for 60 $\mathrm{s}$ (which was the length of both the encoding and retrieval phases). For each list, 12 word pairs were presented auditorily at a pace of one every $5 \mathrm{~s}$ for a total of $60 \mathrm{~s}$ of encoding. Participants then engaged in a 30-s distractor activity in which they had to subtract multiples of 7 from a number that appeared on the screen and write their responses down on a sheet. Participants were told to perform the distractor task as quickly and as accurately as possible. After this interpolated activity, the cued-recall phase began, where participants were given the A word of each pair as a cue and then had to produce the B response within 5-s. This was done for each pair, for a total of 60 -s of retrieval. The order of the cues at retrieval was randomized. Under the full-attention condition, participants were told to pay full attention to the lists in order to encode and retrieve them. In the tracking baseline condition, participants were instructed to catch the asterisk target or to follow it as closely as possible. In the divided-attention conditions they were told to pay equal attention to encoding or retrieval and to the tracking task. Prior to each list, participants were told which attention condition to expect. There were four experimental tasks:

1) Single-task performance: memory full attention (4 trials, two with related and two with unrelated pairs). In this task participants were instructed to encode and retrieve information under full attention conditions.

2) Single-task performance: tracking task (4 trials). Participants performed only the tracking task for $60 \mathrm{~s}$. Each of the trials used one of the four basic paths.

3) Dual-task: divided attention at encoding (4 trials, two with related and two with unrelated pairs). On these trials participants performed the encoding and the tracking task simultaneously, under instructions to pay equal attention to each. In these trials, retrieval was performed under full attention. Each of the trials used one of the four basic paths.

4) Dual-task: divided attention at retrieval (4 trials, two with related and two with unrelated pairs). In each of these trials, participants encoded information under full attention, and then performed the retrieval and the tracking task simultaneously, under instructions to pay equal attention to each. Each of the trials used one of the four basic paths.

The presentation of each word-pair at encoding (via the tape recorder), of the cue word at retrieval, and participants' vocal retrieval of each word, triggered the voice-operated relay which recorded the exact time when each of these events was initiated. Participants initially practiced the tracking task alone, the memory task alone (full attention), and their combination either at encoding (DA at encoding), or at retrieval (DA at retrieval). They then continued with the experimental trials. Twelve formats of order of tasks were used in which the order of the twelve memory trials (four for each attention condition) was counterbalanced using a Latin Square design, and three or four participants were run in each order. The four single tracking task trials were performed before the first list, and after the 4th, 8th and 12th lists. Participants' reports after the experiment indicated that they did not realize that the same four tracking task paths were repeated in the single- and dual-task conditions, but perceived the movement of the asterisk to be random.

\section{Results}

\section{Memory Performance}

Mean percentage of words recalled correctly across trials and participants for each condition appears in Table 1 . A $3 \times 2$ two-way ANOVA with attention condition and pair-relatedness as the two variables, indicated a significant effect of pair relatedness $[\mathrm{F}(1,36)=198.2, \mathrm{p}<0.01$, MSe $=100.0]$, showing that semantically related word pairs $(\mathrm{M}=77.9 \%)$ were better remembered than unrelated pairs $(M=59.0 \%)$. The ANOVA also indicated a significant effect of attention $[\mathrm{F}(2,72)=10.73, \mathrm{p}<$ 0.01 , MSe $=118.4]$. A comparison of full attention $(M=70.9 \%)$ and DA at encoding $(\mathrm{M}=63.6 \%)$ showed a significant difference in performance $[\mathrm{F}(1,36)=15.54, \mathrm{p}<0.01$, MSe $=125.5]$, 
but a comparison of full attention and DA at retrieval $(\mathrm{M}=$ $70.7 \%)$ showed none $[\mathrm{F}(1,64)=<1.0$, ns]. The comparison of $\mathrm{DA}$ at encoding and DA at retrieval was significant $[\mathrm{F}(1,36)=$ $14.98, \mathrm{p}<0.01$, MSe $=1124.2]$. The effect of the interaction of the two variables was not significant $[\mathrm{F}(2,72)=1.03$, ns, MSe $=97.1]$. These results replicate those reported in the literature, in Craik et al., (1996), in Naveh-Benjamin et al. (1998), and in Naveh-Benjamin \& Guez (2000); DA at encoding resulted in a greater drop in memory performance $(7.3 \%$, which is somewhat smaller than the usual one obtained, see discussion below) than did DA at retrieval $(0 \%)$. In terms of memory costs (percentage drop from the full attention condition), the decrease was $11.2 \%$ for encoding and $0 \%$ for retrieval. In this experiment there was no memory cost associated with divided attention at retrieval. These results also replicate the results obtained by NavehBenjamin, Craik \& Ben-Shaul (2002) for word relatedness, showing cued recall to be better for related than for unrelated word pairs.

\section{Retrieval Latency}

For each condition, we averaged the latency for all successful retrievals in each trial (latency for unsuccessful retrievals could not be determined as participants provided no response). Mean latency across trials and participants for each condition appear in Table 1. The mean latency was $1915 \mathrm{~ms}$ in the full attention condition, $2064 \mathrm{~ms}$ in the DA at encoding condition, and 1969 $\mathrm{ms}$ in the DA at retrieval condition. A $3 \times 2$ two-way ANOVA showed a significant effect of attention $[\mathrm{F}(2,72)=8.73, \mathrm{p}<$ 0.01 , MSe $=48415]$. A comparison of full attention with DA at encoding showed a significant difference $([\mathrm{F}(1,36)=18.16$, $\mathrm{p}$ $<0.01$, MSe $=45437]$. A comparison of full attention with DA at retrieval showed a marginally significant effect $[\mathrm{F}(1,36)=$ 2.80, $\mathrm{p}<0.10, \mathrm{MSe}=38889]$. The comparison of the two DA conditions also showed a significant difference $[F(1,36)=5.48$, $\mathrm{p}<0.05$, MSe $=60921]$. These results replicate those obtained in both experiments of Naveh-Benjamin \& Guez (2000), showing that DA both at encoding and at retrieval slowed down retrieval responses, relative to the full attention condition, although

Table 1.

Means and standard deviations (in Parentheses) for the different dependent measures in the different attention conditions for related and unrelated word pairs.

\begin{tabular}{cccc}
\hline & \multicolumn{3}{c}{ Attention Conditions } \\
\hline $\begin{array}{c}\text { Memory (Percentage } \\
\text { Correct) }\end{array}$ & Full Attention & DA-Encoding & DA-Retrieval \\
Related words & $80.0(16.1)$ & $74.4(16.9)$ & $79.2(12.2)$ \\
Unrelated words & $61.8(17.5)$ & $52.9(17.6)$ & $62.2(18.1)$ \\
Latency (in ms) & & & \\
Related words & $1813(221)$ & $1937(251)$ & $1844(216)$ \\
Unrelated words & $2016(261)$ & $2191(373)$ & $2094(292)$ \\
$\begin{array}{c}\text { Tracking-overall } \\
\text { cost (in mm) }\end{array}$ & & & \\
Related words & ----- & $1.14(3.9)$ & $2.42(9.0)$ \\
Unrelated words & ----- & $2.025(5.5)$ & $1.73(8.3)$ \\
\hline
\end{tabular}

in this experiment responses under DA at encoding were slowed down more. The effect of pair relatedness was also significant $[\mathrm{F}(1,36)=46.3, \mathrm{p}<0.01$, MSe $=66867]$, showing slower responses for unrelated $(\mathrm{M}=2100 \mathrm{~ms})$ than for related pairs $(\mathrm{M}=1865 \mathrm{~ms})$. These results provide further evidence for the facilitatory effects of pre-experimental information (semantically related words) in accessing target information. Finally, the interaction of the two variables was not significant $[\mathrm{F}<1.0]$.

\section{Tracking Task Performance}

\section{Retrieval vs. Encoding: Overall Costs}

For each attention condition (except the full one) we averaged the distance (in $\mathrm{mm}$ ) between the target and the tracker after each 50 -ms interval, over the whole trial. To provide a more precise measurement of encoding and retrieval DA costs, we subtracted the appropriate baseline distance (averaged over the 4 baseline trials) for each trial. The resultant overall tracking distances can be seen in Table 1. A $2 \times 2$ two-way ANOVA with attention condition (DA at encoding and at retrieval) and pair relatedness as the two variables, indicated that the effect of attention (mean distance of 1.58 at encoding and 2.08 at retrieval) was not significant $[\mathrm{F}<1.0]$. This result reflects the fact that in a cued-recall task, retrieval does not always require more resources than encoding (e.g., Craik et al., 1996, Experiment 3; Naveh-Benjamin et al., 1998, Experiment 1; Naveh-Benjamin \& Guez, 2000). The results also indicate that both encoding and retrieval required resources for their execution as reflected by the distance measures, which were significantly larger than 0.0 $\mathrm{mm}[\mathrm{t}(36)=2.27$ and 2.50 , for encoding and retrieval respectively; $\mathrm{p}<0.05$ in both cases]. No effect was found for word relatedness $(\mathrm{M}=1.78$ and 1.88, for unrelated and related pairs, respectively), $[\mathrm{F}(1,36)<1.0$, ns, $\mathrm{MSe}=38.35]$. The interaction of the two variables was not significant $[\mathrm{F}<1.0]$. The fact that there are no differences in overall attentional costs between the retrieval of related and unrelated words is somewhat surprising, since we expected semantically related targets to require fewer attentional resources for their retrieval than required for semantically unrelated targets. Such a result indicates that although semantic related targets are recalled faster and with a higher probability than semantic unrelated targets, they require as much attentional resources in this process as semantically related targets. Such a lack of differences in overall attentional costs is not due to the insensitivity of the tracking measure. Naveh-Benjamin \& Guez (2000, Experiment 2) showed that more overall attentional resources are required in the retrieval of infrequent than frequent words.

Since this study was intended not only to compare general resources associated with encoding and retrieval but also to specify the temporal distribution of these resources for encoding and retrieval, we created aggregated 5-s segments for encoding and retrieval, similar to the technique employed in the Naveh-Benjamin \& Guez (2000) and Guez \& Naveh-Benjamin (2006) studies. For encoding, where the presentation of a word pair by the experimenter occurred every $5 \mathrm{~s}$, we superimposed for each participant the 12 five-second segments for each encoding trial, and examined the distribution of tracking performance (after single-task tracking baseline was subtracted). We then averaged performance on the four DA at encoding trials for this participant. For retrieval, the full 5-s segment was used in trials without a retrieval response (unsuccessful retriev- 
als). For trials with a retrieval response (successful retrievals), participants gave their response at different points during the 5-s retrieval interval. Thus, we chose the 2-s mark, as this was the average retrieval latency of the participants (see retrieval latency data above) to represent the retrieval response in the prototypical segment. The next step involved taking all of trials with a retrieval response and dividing each into two segments: from cue presentation to response, and from response to the next cue. We then took each of these two segments for each retrieval interval and superimposed them on the aggregated segment. If the segment cue-response was shorter than $2 \mathrm{~s}$, then the segment was stretched to fit a 2-s interval. Likewise, if the retrieval response took longer than $2 \mathrm{~s}$, the segment was compressed to fit the 2-s interval. Similarly, the intervals from response to cue for each trial were superimposed on the aggregated 3-s interval after the response, by either stretching or compressing them, as necessary. This procedure produced a standardized relative positioning of tracking performance across all words with respect to the periods before or after the actual retrieval.

\section{Retrieval and Encoding Phases.}

In order to analyze the attentional costs associated with different phases of the retrieval process, we broke down the aggregated $5 \mathrm{~s}$ retrieval segment into three components, cue-encoding, cue-elaboration/search, and retrieval mode, as done in the Naveh-Benjamin \& Guez (2000) and Guez \& Naveh-Benjamin (2006) studies. The first component involves the encoding of the cue word, which lasted, on the average, about $500 \mathrm{~ms}$ from the beginning of the mouthing of the cue by the experimenter, based on our measurements which indicated that it took the experimenter about 400 - $500 \mathrm{~ms}$ on the average to say the cue-word (we assume that participants encoded the cue, at least partially, while it was presented auditorily). The second component involves cue-elaboration or search for the appropriate target word, which starts once the cue-word is encoded and ends when the participants overtly retrieve the target word (or a bit earlier, if, when the target is found, participants have to create a motor program in order to mouth the response). As the average retrieval latency was $2000 \mathrm{~ms}$ (see above), the time between 0.5 and $2 \mathrm{~s}$ was designated as cue-elaboration/search time for retrievals. Finally, the time between a successful retrieval and the appearance of the next cue was designated as a retrieval mode only, as participants were presumably not engaged in any active retrieval during this period. To allow for comparison across participants, this segmentation was performed on the adjusted retrieval segments, as described earlier. Note that performance in the baseline single-task tracking condition did not exhibit any particular pattern during the different phases. Furthermore, it did not vary systematically across the different phases.

To isolate the costs associated with each of the above retrieval components and to simplify the analysis, we used the first data point in Table 2 ( 0.0 to $0.5 \mathrm{~s})$ to estimate the attentional cost associated with cue-encoding, the mean of the second, third, and fourth data points $(0.5-1.0$ to $1.5-2.0 \mathrm{~s})$ to estimate the cost associated with cue-elaboration/search, and the mean of the sixth, seventh, eighth, and ninth data points (2.5 - 3.0 to $4.0-4.5$ s) to estimate the cost associated with retrieval mode. In this analysis we did not include either the fifth data point $(2.0-2.5)$, since in some of the trials it was associated
Table 2.

Secondary tracking task means and standard deviations (in Parentheses) for the different phases of the encoding and retrieval segments of related and unrelated word pairs (Distance in $\mathrm{mm}$ ).

\begin{tabular}{|c|c|c|c|}
\hline & \multicolumn{3}{|c|}{ Phase } \\
\hline & Cue-encoding* & $\begin{array}{l}\text { Cue-Elaboration/ } \\
\text { Search* }\end{array}$ & Mode \\
\hline \multicolumn{4}{|l|}{ Encoding } \\
\hline Related words & 0.72 (3.7) & 0.92 (3.7) & $1.31(4.2)$ \\
\hline $\begin{array}{l}\text { Unrelated } \\
\text { words }\end{array}$ & $1.51(5.5)$ & $1.98(5.6)$ & $2.14(5.6)$ \\
\hline \multicolumn{4}{|l|}{ Retrieval } \\
\hline Related words & $2.01(8.1)$ & 3.35 (8.9) & $1.84(9.2)$ \\
\hline $\begin{array}{l}\text { Unrelated } \\
\text { words }\end{array}$ & 1.49 (7.4) & 2.64 (7.9) & $1.03(8.7)$ \\
\hline \multicolumn{4}{|l|}{$\begin{array}{l}\text { Unsuccessful } \\
\text { retrievals }\end{array}$} \\
\hline Related words & $3.96(12.5)$ & $5.38(12.4)$ & $5.61(11.8)$ \\
\hline $\begin{array}{l}\text { Unrelated } \\
\text { words }\end{array}$ & $1.30(8.5)$ & $2.90(8.4)$ & $3.31(10.0)$ \\
\hline \multicolumn{4}{|l|}{$\begin{array}{l}\text { Successful } \\
\text { retrievals }\end{array}$} \\
\hline Related words & $1.62(7.3)$ & $3.01(8.5)$ & 0.97 (8.6) \\
\hline $\begin{array}{l}\text { Unrelated } \\
\text { words }\end{array}$ & $1.38(7.3)$ & $2.42(7.8)$ & $-0.26(8.3)$ \\
\hline \multicolumn{4}{|l|}{$\begin{array}{c}\text { Slow } \\
\text { retrievals }\end{array}$} \\
\hline $\begin{array}{l}\text { Related } \\
\text { words }\end{array}$ & $1.48(7.8)$ & $3.42(9.2)$ & $0.54(9.2)$ \\
\hline $\begin{array}{l}\text { Unrelated } \\
\text { words }\end{array}$ & $2.13(8.3)$ & $3.52(9.0)$ & $0.08(9.2)$ \\
\hline \multicolumn{4}{|l|}{$\begin{array}{c}\text { Fast } \\
\text { retrievals }\end{array}$} \\
\hline Related words & $1.26(7.2)$ & $1.93(7.7)$ & $1.21(8.2$ \\
\hline $\begin{array}{l}\text { Unrelated } \\
\text { words }\end{array}$ & $-0.58(6.6)$ & $-0.46(6.4)$ & $-0.52(7.4)$ \\
\hline
\end{tabular}

with retrievals, or the tenth data point (4.5 - 5.0), since its results may be confounded with anticipation of the next cue-word. For comparison purposes only, we similarly designated the respective periods during encoding, as stimulus encoding, encoding processes, and encoding mode. This division (a fairly arbitrary one) is based on the segmentation at retrieval. For example, it would have been equally possible to have the encoding processes phase occupy the entire period after the stimuli were encoded rather than being followed by an encoding mode phase (see General Discussion). The resultant means for unrelated and semantically related pairs appear in Table 2. Several patterns can be observed in Table 2. First, as already mentioned above, both encoding and retrieval required attentional resources for their execution, as reflected by the distance function being above the baseline $(0.0 \mathrm{~mm})$. Second, there seems to be an effect of phase. Specifically, the cue-elabora- tion/search phase seems to require more resources than the cue-encoding 
and mode phases. The table also indicates an interaction between encoding/retrieval and phase, where the difference between encoding and retrieval is the greatest in the cue-elaboration/search phase. A $2 \times 2 \times 3$ three-way ANOVA with attention condition (DA at encoding and at retrieval), pair relatedness, and phase-component as the three variables indicated no significant effect of encoding vs. retrieval $[F<1.0]$, no significant effect of pair relatedness $[\mathrm{F}<1.0]$, and a significant effect of phase-component $[\mathrm{F}(2,72)=8.30, \mathrm{p}<0.01$, MSe $=$ 3.25]. Follow-up analyses showed that the significant effect of phase-component was due to the greater resources required in the cue-elaboration/search phase than in the cue-encoding and mode phases $([\mathrm{F}(1,36)=14.14, \mathrm{p}<0.001, \mathrm{MSe}=3.38]$, and $[\mathrm{F}(1,36)=12.64, \mathrm{p}<0.01, \mathrm{MSe}=2.48]$, respectievly). The only significant interaction was that of encoding/retrieval and phase component $[\mathrm{F}(2,74)=11.26, \mathrm{p}<0.01$, $\mathrm{MSe}=2.84]$. Several follow-up analyses were performed on this significant interaction. Analysis of simple effects of the phase-component for encoding indicated significant differences $[(F(2,72)=3.43$, $\mathrm{p}<0.05$, MSe $=1.03$ ]. Follow-up comparisons for encoding indicated one significant difference, between the cue-encoding and the mode phase $[\mathrm{F}(1,36)=5.14, \mathrm{p}<0.05$, MSe $=1.37]$, where the latter was associated with larger distance. A similar analysis for retrieval also indicated significant differences $[\mathrm{F}(2$, $72)=12.88, \mathrm{p}<0.00, \mathrm{MSe}=2.01]$. Follow-up comparisons for retrieval indicated that the distance measure was larger in the cue-elaboration/search phase than in the cue-encoding and the mode phases $([\mathrm{F}(1,36)=13.81, \mathrm{p}<0.00$, MSe $=2.15]$, and $[\mathrm{F}(1,36)=31.1, \mathrm{p}<0.00, \mathrm{MSe}=1.48]$, respectively). The latter two were not different from each other $[\mathrm{F}<1.0]$. These results replicate those obtained in Naveh-Benjamin \& Guez (2000, Experiment 2).

\section{Types of Retrieval}

To specify the attentional costs associated with unsuccessful retrievals, we separated (as in Naveh-Benjamin \& Guez, 2000), trials in which participants provided no retrieved response (designated unsuccessful retrievals), from those where a retrieved response was given (designated successful retrievals, since in over $95 \%$ of the cases when a word was retrieved, it was the correct one; we therefore took all responses to indicate successful retrievals). Furthermore, to assess potential differences among the successful retrievals (see Naveh-Benjamin \& Guez, 2000) we split them, as in Experiment 1, into two groups: fast retrievals comprising those retrievals with response latency of $1400 \mathrm{~ms}$ or less (mean = $1125 \mathrm{~ms}$ ), and slow retrievals, comprising those retrievals with a response latency over 1400 ms (mean latency $2356 \mathrm{~ms}$ ). This resulted in $25 \%$ out of all successful retrievals being fast and $75 \%$ being slow. We created two aggregated retrieval segments, one for fast retrievals and one for slow retrievals. In order to analyze the attentional costs associated with different phases of the retrieval period for unsuccessful, slow, and fast retrievals, we broke down the 5-s retrieval period into the three components mentioned above -cue-encoding, cue-elaboration/search, and retrieval mode. Table 2 presents secondary task tracking performance averaged across all participants (distance in $\mathrm{mm}$ ) for the aggregated 5-s unsuccessful, slow successful, and fast successful retrieval segments for the related and unrelated pairs separately, after single-task tracking performance was subtracted. This secondary task performance represents the overall temporal distribu- tion of attentional costs associated with each type of retrieval. The distribution of the distance measure for each type of retrieval across time can be seen in Figure 1. The encoding component was added for completeness.

A $3 \times 2 \times 3$ three-way ANOVA with retrieval type as one variable, pair relatedness as the other variable, and phase/ component as the third variable, indicated a significant effect of type of retrieval $[\mathrm{F}(2,72)=8.63, \mathrm{p}<0.01$, MSe $=69.44]$ (see Table 2). Further comparisons indicated that fast retrievals (M $=0.47)$ were associated with shorter distance than either slow retrievals $(\mathrm{M}=1.86)$, or unsuccessful ones $(\mathrm{M}=3.75),[\mathrm{F}(1,36)$ $=3.52, \mathrm{p}=0.06]$, and $[\mathrm{F}(1,36)=11.53, \mathrm{p}<0.01]$, respectively. In addition, slow retrievals were associated with shorter distance than unsuccessful ones $[\mathrm{F}(1,36)=8.96, \mathrm{p}<0.01]$. Also, the effect of component/phase was significant $[\mathrm{F}(2,72)=7.44$, $\mathrm{p}<0.01$, MSe $=12.89]$. Further comparisons showed that the search/cue-elaboration phase $(M=2.78)$ was more demanding than either the cue-encoding ( $M=1.59)$ or the mode $(M=1.70)$ phases $[\mathrm{F}(1,36)=11.25, \mathrm{p}<0.01$, MSe $=14.0]$, and $[\mathrm{F}(1$, 36) $=15.62, \mathrm{p}<0.01$, MSe $=8.23$ ], respectively. The latter two phases were not different from each other $[\mathrm{F}<0.01]$. Finally, the effect of pair relatedness was not significant $[F<1.0]$, indicating that the distance measure for unrelated pairs was not different than the one for related ones. Most importantly, the interaction of type of retrieval and component/phase was significant $[\mathrm{F}(4,144)=10.45 ; \mathrm{p}<.01$, MSe $=7.83]$ (see Table 2). Several follow-up comparisons were carried out. The first one comparing unsuccessful and slow successful retrievals in the cue-encoding and cue-elaboration search phases showed no significant interaction $[F<1.0]$. This reflects the fact that both retrievals showed the same increase in the distance measure from the cue-encoding to the cue-elaboration phase. In contrast, these types of retrieval show an interaction with phase/component, when cue-elaboration and retrieval mode phases are inspected $[\mathrm{F}(1,36)=25.9, \mathrm{p}<.01$, MSe $=8.65]$. This interaction reflects the decrease in distance from the cue-elaboration/search to the retrieval mode phase in the slow retrievals, coupled with no such a decrease in the unsuccessful retrievals. Such a pattern of results for the unsuccessful retrieval is consistent with the notion that participants continuously allocate attention in an attempt to retrieve the target. The pattern of results for slow

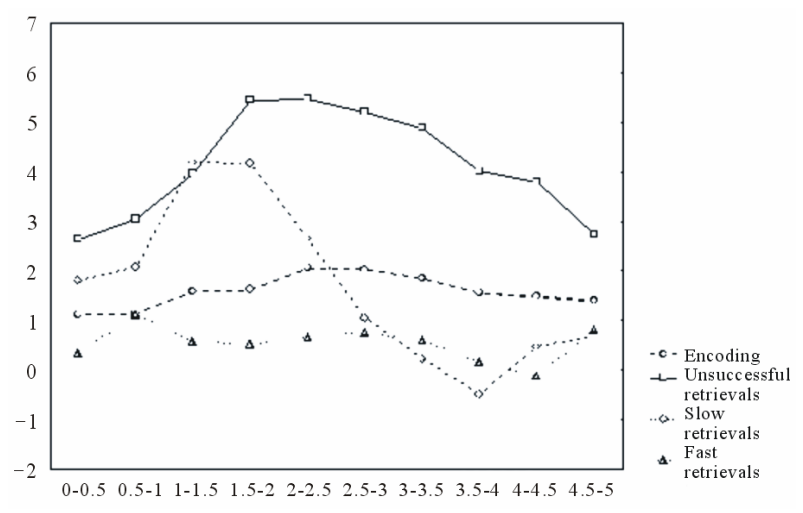

Figure 1.

Temporal distribution of performance on the secondary track- $g$ task for the aggregated 5-s encoding, unsuccessful retrieval, slow retrieval, and fast retrieval segments after single-task tracking performance subtraction (distance in millimeters). 
retrievals is consistent with the idea that greater attentional resources are allocated to retrieve the target, and then fewer are allocated once the target was retrieved. The next interaction comparison looked at slow and fast retrievals in the cue-encoding and cue-elaboration/search phases. This effect was significant $[\mathrm{F}(1,36)=9.16, \mathrm{p}<0.01$, MSe $=3.26]$ (see Table 2 and Figure 2(a)). It reflects the fact that slow retrievals showed an increase in the distance measure from cue-encoding to cue-elaboration/search $[\mathrm{F}(1,36)=17.69, \mathrm{p}<0.01$, $\mathrm{MSe}=5.81]$, whereas fast retrievals did not $[\mathrm{F}<1.0]$. In addition, the interaction of slow and fast retrievals and the cue-elaboration and retrieval mode components was significant $[\mathrm{F}(1,36)=23.9, \mathrm{p}<$ 0.01 , MSe $=5.93]$, reflecting the fact that for slow retrievals there was a decrease in the distance measure from the cue-elaboration/search to the retrieval mode component $[\mathrm{F}(1$, $36)=41.84, \mathrm{p}<0.01, \mathrm{MSe}=8.84]$; fast retrievals did not show this trend $[\mathrm{F}<1.0]$. Finally, follow up interaction comparisons of unsuccessful and fast retrievals showed an interaction which approached significance $[\mathrm{F}(1,36)=3.76, \mathrm{p}=.06$, $\mathrm{MSe}=6.11]$, reflecting the increased distance from the cue-encoding to cue-elaboration/search phase for unsuccessful retrievals $[\mathrm{F}(1$, 36) $=8.67, \mathrm{p}<0.01$, MSe $=9.74]$, but no such increase in the fast retrievals $[F<1.0]$. The unsuccessful and fast retrievals did not interact with the cue-elaboration retrieval mode phases, suggesting that both continued to require the same amount of attention resources in the retrieval mode phase as in the cueelaboration/search one $[\mathrm{F}(1,36)=1.14$, ns., MSe $=8.06]$. All other effects were not significant $[\mathrm{F}<1.0$ in all cases]. In particular, unrelated and related pairs demonstrated similar patterns of attentional resource allocation (see Figure 2(b)). The above analyses reveal a substantial increase in attentional resources associated with cue-elaboration/search processes, along with a decrease in these resources once retrieval is successful. This pattern was observed for both unsuccessful and slow retrievals. For unsuccessful retrievals, a continuous search for the target was associated with substantial attentional resources being required after cue-encoding, and throughout the retrieval period. Fast retrievals, on the other hand, did not show this increase in attentional resources throughout the cue-elaboration/ search phase. Interestingly, these patterns characterize both related and unrelated word pairs. The different phases of retrieval of semantically related and unrelated targets seem to be similar in terms of the attentional costs required. This result is somewhat unexpected since we were expecting the semantic relationships between the cue and the target to decrease the amount of attentional resources required for the cue-elaboration processes. Pre-experimental knowledge seems to affect both the rate of access to the information (reflected by retrieval latency), and its success (reflected by memory accuracy), but does not affect the attentional resources associated with this retrieval. It seems, as with similar results obtained by Naveh-Benjamin \& Guez (2000) with low- and high-frequency words, that the increase in attentional requirements involved in cue-elaboration/ search of the target word is constant and not necessarily related to the type of materials used.

\section{Proportion of Slow and Fast Retrievals}

We looked at the proportion of fast and slow retrievals in the related and unrelated pairs. Mean percentage of fast retrievals of all successful retrievals was $35.4 \%$ in the related pairs condition and $22.9 \%$ in the unrelated pair condition. A t-test showed

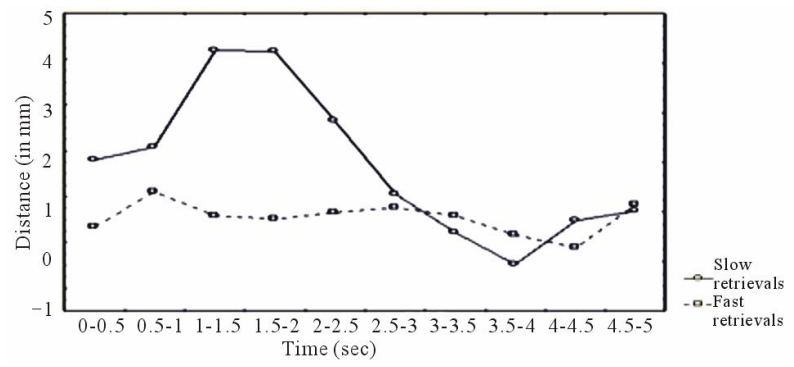

(a)

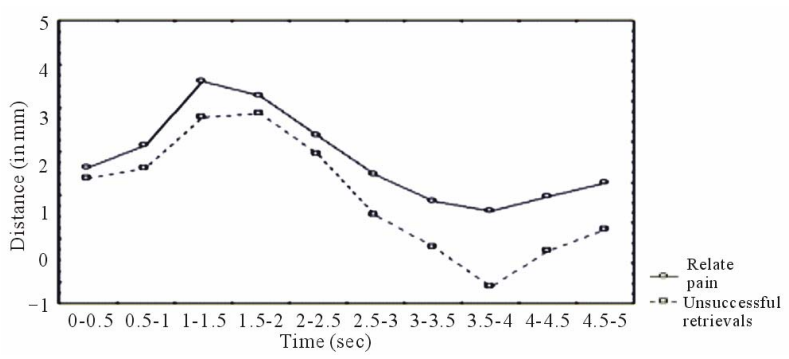

(b)

Figure 2.

(a). Temporal distribution of performance on the secondary tracking task for the aggregated 5-s successful retrieval segments after single-task tracking performance subtraction (distance in millimeters), for slow and fast retrievals. (b). Temporal distribution of performance on the secondary tracking task for the aggregated 5-s successful retrieval segments after single-task tracking performance subtraction (distance in millimeters), for semantically related and unrelated pairs.

that these proportions were significantly different from each other $[\mathrm{t}(36)=6.40, \mathrm{p}<0.01]$. These results indicate that the better memory performance in the related pairs condition could be due to the increase in the proportion of fast retrievals in this condition relative to the unrelated pairs condition.

\section{Discussion}

Overall, the results of this experiment replicated and extended some recent results reported by Naveh-Benjamin \& Guez (2000) and Guez \& Naveh-Benjamin (2006). First, DA at encoding, but not at retrieval, affected memory performance. In addition, retrieval latency was slowed down under DA both at encoding and at retrieval. Also, the attentional costs associated with retrieval were mostly related to the time after the cue was encoded and before a retrieval response was initiated. Successful retrievals show this pattern until a retrieval response is produced, whereas unsuccessful retrieval show it throughout the retrieval period until the next cue is presented. Furthermore, successful retrievals appear in two distinct varieties: slow and fast. Whereas the former are associated with increase in resources during the cue-elaboration/search phase, the latter show no increase in resources associated with search for the target. The results of the manipulation of pair relatedness yield similar patterns to those obtained in the authors previous reports in showing, again, the resilience of retrieval process to the effects of divided attention. However, while related pairs seem to be retrieved faster and with a higher success rate, the attentional cost to achieve this retrieval is not different than that for unre- 
lated pairs. In particular, retrieving a target which is semantically related to the cue seems to require attention during the cue-elaboration/search processes to the same degree as when the target is unrelated semantically to the cue. Pre-experimental knowledge seems to help in rate of access and its success but does not reduce the effort required.The outcome of the current experiment converges on results reported in the past, and, in addition, supplies further information about the effects of divided attention on retrieval processes, and the attentional costs associated with these processes. The current experiment replicated previous results in showing an asymmetry between encoding and retrieval processes under the effects of divided attention: whereas dividing attention at encoding significantly downgrades memory performance, dividing attention at retrieval has almost no effect on memory performance. This was demonstrated in the reported experiment, using a tracking task which served as the secondary task, and cued-recall as the memory task. Retrieval processes, however, required substantial attentional resources as reflected by performance on the secondary tracking task. In the first glance, comparing encoding and retrieval overall cost it seems that the resources required during retrieval were the same than those required during encoding. These patterns are similar to those reported in the literature for cued-recall tasks. While some studies show retrieval to require overall more resources than encoding (e.g., Craik et al., 1996, Ex. 1 and 2; Naveh-Benjamin et al., 1998; NavehBenjamin \& Guez, 2000, Experiment 1), others (Craik et al., 1996, Ex. 3; Naveh-Benjamin, et al., 1998, Experiment 2, Naveh-Benjamin, et al., 2000a; Naveh-Benjamin \& Guez, 2000, Experiment 2), show the two to require substantial but equal resources. The nature of the secondary task can not explain the different patterns of attentional costs at encoding and retrieval, as the same tracking task was used Naveh-Benjamin \& Guez (2000) and Guez \& Naveh-Benjamin (2006) experiments. Interestingly, free recall is associated with larger attentional costs than encoding (Craik et. al., 1996, Anderson, Craik \& NavehBenjamin, 1998). Yet, in our suggested view this general comparison and its variety needs more closer analysis in order to test which type or phase take what amount of resource.

\section{Phases of Retrieval}

One purpose of the research reported here was to further assess the attentional costs associated with retrieval. For this purpose, the current study included a cued-recall task which enables one the separation of the attentional resources required. Based partially on Tulving's (1983) taxonomy of retrieval sub-processes, and on results by Naveh-Benjamin \& Guez (2000) and Guez \& Naveh-Benjamin (2006), we have designated three retrieval phases or subcomponents: cue encoding, cue-elaboration/search processes for a specific word, and the operation of a retrieval mode. The results indicate, as in Naveh-Benjamin \& Guez (2000), but in contrast to previous studies, that retrieval, at least during a cued-recall task, does not require uniform attentional resources. The first phase of cue-encoding requires some resources, much like stimuli detection requires at encoding. The second phase, involving cue-elaboration/search processes requires substantial resources for its execution. This was shown in two ways. First, for successful retrievals, the period after the cue-encoding and prior to the retrieved response was associated with poorer performance on the secondary task (larger tracking distance), than either the respective period at encoding or the retrieval period following the retrieval response. Second, the use of a cued-recall task allowed us to measure attentional resources associated with both successful and unsuccessful retrievals. Performance on the secondary task for unsuccessful retrievals (where no retrieved response was provided by the participant) was poor throughout the retrieval period of a given target. Specifically, while unsuccessful retrievals consume attentional resources for their execution as successful retrievals do up to the point of the retrieved response, these unsuccessful retrievals continue to require resources for the whole retrieval period, presumably reflecting the continuation of cue-elaboration/search processes. With respect to the question of whether being in a retrieval state of mind which operates in the background (retrieval mode), also requires attentional resources, the answer is not clear-cut. When this is evaluated by looking at successful retrievals after their completion-a period when participants were not trying to retrieve any given word (they did not know which cue would be presented next) - the attentional costs were not significantly higher than the baseline. This finding is not in line with our previews finding showing retrieval mode requiring attentional costs above and beyond those required in the secondary task baseline condition (Naveh-Benjamin \& Guez (2000) and Guez \& Naveh-Benjamin (2006).

\section{Types of Retrieval}

Successful and unsuccessful retrievals showed both similarities and differences. While both require the same amount of attentional resources for the execution of the cue-encoding and cue-elaboration phases, after these phases the pattern of their attentional cost diverges: whereas successful retrievals (where the retrieval response terminates the cue-elaboration/search phase moving participants to a retrieval mode phase), are associated with fewer attentional resources, unsuccessful retrievals (where the cue-elaboration/search phase proceeds until the next cue is presented), are associated with continuous high attentional costs2. These results obtained here, replicate and extend those reported by the authors in the context of other manipulations. Another issue studied in the reported experiment concerned the existence of different types of successful retrievals. We wanted to find out whether different types of retrievals can be distinguished and their associated attentional costs be measured. One measure used here to distinguish these types of retrieval is of retrieval latency; we distinguished slow from fast retrievals and estimated the attentional resources associated with each. Results indicated that these two types of retrievals require different amount of resources for their execution. Slow retrievals were associated with substantial attentional resources for their execution, especially during the cue-elaboration/search phase. Fast retrievals, however, required fewer resources for their execution, and in particular did not seem to show an increase in required attentional resources during the cue-elaboration/search phase. One way to interpret these results is by suggesting that fast retrievals bypass and do not require cue- elaboration/search processes. Using such an interpretation, the current work suggests the operation of two qualitatively different types of successful retrievals: one which is fast and requires few attentional resources for its completion (one-step), and the other which is slower and requires substantial resources 
(strategic). Note that the measurements of retrieval latency and secondary task performance were independent of each other. Fast retrievals could therefore theoretically require less, more, or the same amount of attentional resources as their slower counterparts. Interestingly, the semantic relatedness manipulation of the cue to the target did not affect the resource allocation for slow and fast retrievals. In particular, it did not affect the attentional patterns during the cue-elaboration/search phase; that is, the attentional resources associated with the processes involved in searching for the target in an episodic memory task do not seem to be sensitive to the pre-experimental semantic relationships between the cue and the target. The search for the target involves the same amount of attentional resources regardless of whether the cue is related or unrelated to the target, even though a related cue increases the probability and the speed at which the target is episodically retrieved. The lack of differences in the attentional costs associated with the retrieval of semantically related and unrelated words is somewhat surprising. It may be explained by the fact that in the related-pairs condition, the cue provided information about the target, but since not all the targets were the strongest possible ones associated semantically with the cue, on some occasions, another strong pre-experimentally related association was retrieved. It forced the participants to inhibit this potent response and to continue their search for the correct target, resulting in extra effort associated with the cue-elaboration/search phase of the strongly related targets. These results are somewhat different from Guez \& Naveh-Benjamin (2006) using repetition manipulation. It seem that providing episodic enhancement to the cue-target relationships, by repeating word pairs three times during the study phase, increases the probability of retrieval success and the speed of the retrieval. In addition, in contrast to the current report, episodic enhancement at encoding leads to retrieval which requires less attentional resources. In particular, fewer resources are required in this case during the cue-elaboration/search component. Interestingly, this effect seems to be mediated by a change in the nature of retrievals of the thrice- presented pairs; the retrieval of these pairs seems to mostly bypass the search process, making it faster and less dependent on attentional resources.

As mentioned in the Introduction, the identification of two types of successful retrievals is in line with several suggestions in the literature that the retrieval process is not unitary and that different retrievals may be based on different mechanisms. For example, Jacoby $(1991 ; 1998)$ distinguishes automatic retrievals based on familiarity from controlled ones based on recollection. Gardiner and Java (1993) distinguish retrievals based on recollective experience (remember responses) from ones based on familiarity only (know responses). Although both Jacoby and Gardiner and Java use these terms mostly within the context of a recognition paradigm, it seems plausible that the one-step retrievals we have witnessed could be related to those retrievals which are automatic, according to Jacoby (though controlled retrieval also can sometimes be faster, too), or to those which Gardiner and Java suggest are based on familiarity. Our strategic retrievals may be related to those retrievals termed controlled by Jacoby or to those based on remember responses as suggested by Gardiner and Java. Further research should address this apparent similarity. Note that while the distinction between controlled and automatic retrievals, made by Jacoby and Gardiner and Java, is based on subjective responses by the participants, our current results extend these by providing objective indices of latency, and resources associated with each type of retrieval.

\section{Employment of a Multi-Measure Approach}

The current results, along with those reported by the authors (2000, 2006), suggest that a thorough investigation of encoding and retrieval processes should involve several measures of performance that allow, separately and in combination, the uncovering of different facets and components of these processes. While previous studies used either a uni-measure approach (e.g., Murdock, 1965—only accuracy; Johnston, et al., 1970—only overall attentional costs; Carrier \& Pashler, 1995-only latency) or a bi-measure approach (e.g., Baddeley, et al., 1984-accuracy and latency; Craik, et al., 1996, and Naveh-Benjamin et al., 1998-accuracy and attentional costs), we suggest the use of multi-measure approach. In the current study we used four measures of performance: memory accuracy, retrieval latency, overall attentional costs, and the temporal distribution of attentional costs. We believe that such an approach, provides a more comprehensive perspective on the phenomenon under investigation. In particular, the introduction of on-line temporal measurements of a specific pattern of behavior could help us in reaching conclusions regarding characteristic processes which, in combination, yield that behavior. The current approach is related to a line of research on retrieval processes, which tries to unveil the dynamics of memory retrieval. Several studies (e.g., Hintzman \& Curren, 1994, Hintzman \& Caulton, 1997; Dosher, 1981, McElree \& Dosher, 1993) have used variations on the response-signal method to determine how the retrieval of a single item or event unfolds over time. In this method, the amount of time available for retrieval is varied and memory accuracy is plotted as a function of the increase in processing time. Although the studies reported in the current article were run within a different research context and for a different purpose, and have, moreover, employed a cued-recall procedure rather than one based on recognition, the two approaches have some aspects in common. In particular, whereas the responsesignal procedure focuses on the time-related dynamics of memory accuracy, the current study focus on the dynamics of the attentional resources associated with memory accuracy. It may be interesting to find out whether the different retrieval dynamics patterns established for different memory tasks within the response signal procedure also show different attentional patterns. The results of the current experiment indicate that there may be both similarities and differences in the results obtained by each procedure. For example, whereas Dosher \& Rosedale (1991) showed that the rate at which information is retrieved is faster when pre-experimental associations, in addition to episodic ones, are employed (consistent with our latency results), our current results show similar attentional resources patterns associated with the retrieval of these different types of associations.

\section{Conclusion}

The current research extends the conclusions of our earlier research by indicating that the processes associated with encoding and retrieval processes are different. While divided attention at encoding interferes markedly with later memory 
performance divided attention at retrieval does so to a much lesser degree. Retrieval processes, however, seem to be as attention-demanding as encoding processes or even more so. This demand for attentional resources is related to whether retrieval was successful or not, and whether it is strategic or direct. In addition, the current study indicated that this demand for resources is modulated by the retrieval phase, where cue-elaboration/search processes, in particular, require substantial resources for their execution.

\section{References}

Anderson, N. D., Craik, F. I. M., \& Naveh-Benjamin, M. (1998). The attentional demands of encoding and retrieval in younger and older adults: I. Evidence from divided attention costs. Psychology and Aging, 13, 405-423. doi:10.1037/0882-7974.13.3.405

Baddeley, A. D., Lewis, V., Eldridge, M., \& Thomson, N. (1984). Attention and retrieval from long-term memory. Journal of Experimental Psychology: General, 13, 518-540. doi:10.1037/0096-3445.113.4.518

Balgur, R. (1968). List of basic words for school. Israel: Otsar Hamoreh.

Bransford, J. D., \& Johnson, M. K. (1972). Contextual prerequisites for understanding: Some investigations of comprehension and recall. Journal of Verbal Learning and Verbal Behavior, 11, 717-726. doi:10.1016/S0022-5371(72)80006-9

Carrier, M. L., \& Pashler, H. (1995). Attentional limits in memory retrieval. Journal of Experimental Psychology: Learning, Memory, and Cognition, 21, 1339-1348. doi:10.1037/0278-7393.21.5.1339

Craik, F. I. M., Govoni, R., Naveh-Benjamin, M., \& Anderson (1996). The effects of divided attention on encoding and retrieval processes in human memory. Journal of Experimental Psychology: General, 125, 159-180. doi:10.1037/0096-3445.125.2.159

Dosher, B. A. (1981). The effects of delay and interference: A speed-accuracy study. Cognitive Psychology, 13, 551-582. doi:10.1016/0010-0285(81)90020-7

Dosher, B. A. (1984). Discriminating preexperimental (semantic) from learned (episodic)associations: A speed-accuracy study. Cognitive Psychology, 16, 519-555. doi:10.1016/0010-0285(84)90019-7

Dosher, B. A., \& Rosedale, G. (1991). Judgments of semantic and episodic relatedness: Common time-course and failure of segregation. Journal of Memory and Language, 30, 125-160. doi:10.1016/0749-596X(91)90001-Z

Danker, J. D. \& Anderson, J. R. (2010). The Ghosts of Brain States Past: Remembering Reactivates the Brain Regions Engaged During Encoding. Psychological Bulletin, 136, 87-102. doi:10.1037/a0017937

Gardiner, J. M., and Java, R. I. (1993). Recognizing and remembering. In A. F. Collins, S. E. Gathercole, M. A. Conway, \& P. E. Morris (Eds.). Theories of Memory,. Hove, UK: Erlbaum.

Griffith, D. (1976). The attentional demands of mnemonic control processes. Memory and Cognition, 4, 103-108. doi:10.3758/BF03213261

Guez, J. \& Naveh-Benjamin, M. (2006). Divided Attention at Encoding and Retrieval for Once- and Thrice-Presented Items: A Micro-Level Analysis of Attentional Costs. European Journal of Cognitive Psychology, 18 (6), 874-898. doi:10.1080/09541440500485854

Guynn, M. J., \& McDaniel, M. A. (1999). Generate - Sometimes recognized, sometimes not. Journal of Memory and Language, 41, 398-415. doi:10.1006/jmla.1999.2652

Henik, A., \& Kaplan, L. (1988). Categories content: Findings regarding Hebrew categories and comparison to U.S. finding. Psychology, 1, 104-112.

Herrmann, D. J., \& McGuighlan, J. P. (1974). Recognition latency for a subjectively organized list. Journal of Experimental Psychology, 102, 888-889. doi:10.1037/h0036342

Hintzman, D. L., \& Curran, T. (1994). Retrieval dynamics of recogni- tion and frequency judgments: Evidence for separate processes of familiarity and recall. Journal of Memory and Language, 33, 1-18. doi:10.1006/jmla.1994.1001

Hintzman, D. L., \& Caulton, D. A. (1997). Recognition memory and modality judgments: A comparison of retrieval dynamics. Journal of Memory and Language, 37, 1-23. doi:10.1006/jmla.1997.2511

Jacoby, L. L. (1991). A process dissociation framework: Separating automatic from intentional uses of memory. Journal of Memory and Language, 30, 513-541. doi:10.1016/0749-596X(91)90025-F

Jacoby, L. L. (1998). Invariance in automatic influences of memory: Toward a user's guide for the process-dissociation procedure. Journal of Experimental Psychology: Learning, Memory, and Cognition, 24, 3-26. doi:10.1037/0278-7393.24.1.3

Johnston, W. A., Greenberg, S. N., Fisher, R. P., \& Martin, D. W. (1970). Divided attention: A vehicle for monitoring memory processes. Journal of Experimental Psychology, 83, 164-171. doi:10.1037/h0028554

Johnston, W. A., Griffith, D., \& Wagstaff, R. R. (1972). Speed, accuracy, and ease of recall. Journal of Verbal Learning and Verbal Behavior, 11, 512-520. doi:10.1016/S0022-5371(72)80034-3

Johnston, W. A., \& Uhl, C. N. (1976). The contributions of encoding effort and variability to the spacing effect on free recall. Journal of Experimental Psychology: Learning, Memory, and Cognition, 2, 153-160.

Kolers, P. A. (1973). Remembering operations. Memory and Cognition, 1, 347-355. doi:10.3758/BF03198119

McElree, B., \& Dosher, B.A. (1993). Serial retrieval processes in the recovery of order information. Journal of Experimental Psychology: General, 122, 291-315. doi:10.1037/0096-3445.122.3.291

Mishkin, M., \& Appenzeller, T. (1987). The anatomy of memory. Scientific American, 256, 80-89. doi:10.1038/scientificamerican0687-80

Morris, C. D., Bransford, J. D., \& Franks, J. J. (1977). Levels of processing versus transfer appropriate processing. Journal of Verbal Learning and Verbal Behavior, 16, 519-533. doi:10.1016/S0022-5371(77)80016-9

Moscovitch, M. (1992). Memory and working-with-memory: A component process model based on modules and central systems. Journal of Cognitive Neuroscience, 4, 257-267. doi:10.1162/jocn.1992.4.3.257

Murdock, B. B. Jr. (1965). Effects of a subsidiary task on short-term memory. British Journal of Psychology, 56, 413-419. doi:10.1111/j.2044-8295.1965.tb00983.x

Naveh-Benjamin, M., Craik, F. I. M., \& Ben-Shaul, L. (2002). Agerelated differences in cued recall: Effects of support at encoding and retrieval. Aging, Neuropsychology \& Cognition, 9, 276-287. doi:10.1037/0278-7393.24.5.1091

Naveh-Benjamin, M., Craik, F. I. M., Guez, J. \& Dori, H. (1998). Effects of divided attention on encoding and retrieval processes in human memory: Further support for an asymmetry. Journal of Experimental Psychology: Learning, Memory and Cognition, 24, 1091-1104. doi:10.3758/BF03209344

Naveh-Benjamin, M., Craik, F. I. M., Gavrilescu, D., \& Anderson, N. D. (2000a). Asymmetry between encoding and retrieval processes: Evidence from divided attention and a calibration analysis. Memory and Cognition, 28, 965-976. doi:10.1080/027249800410454

Naveh-Benjamin, M., Craik, F. I. M., Perretta, J. G., \& Tonev, S. T. (2000b). The effects of divided attention on encoding and retrieval processes: The resiliency of retrieval processes. The Quarterly Journal of Experimental Psychology, 53, 609-625. doi:10.1080/027249800410454

Naveh-Benjamin, M., \& Guez, J. (2000). Effects of divided attention on encoding and retrieval processes: Assessment of attentional costs and a componential analysis. Journal of Experimental Psychology: Learning, Memory and Cognition, 26, 1461-1482. doi:10.1037/0278-7393.26.6.1461

Roediger, H. L. (1990). Implicit memory: Retention without remembering. American Psychologist, 45, 1043-1056. doi:10.1037/0003-066X.45.9.1043

Roediger, H. L., Gallo, D. A., \& Geraci, L. (2002). Processing ap- 
proaches to cognition: The impetus from the levels-of-processing framework. Memory, 10, 319-332. doi:10.1080/09658210224000144

Squire, L. R. (1992). Memory and brain. New York: Oxford University Press.

Tulving, E., \& Thomson, D. M. (1973). Encoding specificity and re- trieval processes in episodic memory. Psychological Review, 80, 352-373. doi:10.1037/h0020071

Tulving, E. (1983). Elements of episodic memory. New York: Oxford University Press. 